\title{
Custom Unicompartmental Knee Arthroplasty
}

\author{
Etienne L. Belzile, Michèle Angers, \\ and Martin Bédard
}

\section{Key Points}

- Modern medicine now involves decreasing cost of surgical intervention while improving patient function and outcome.

- Renewed focus in kinematic alignment of the knee brought back the concept of minimizing biomechanical modifications during knee arthroplasty.

- A custom design UKA shows promising strategies exploiting knowledge from past designs and positioning its expected development in line with existing variability in functional knee phenotypes.

\subsection{Introduction}

Customization of a unicompartmental knee arthroplasty (UKA) implant is a new surgical philosophy aiming at reproducing the patient's anatomy and joint morphology while minimizing modifications in the biomechanics of the knee joint during reconstruction. Combining the advantages of single-use patient-specific instrumentation

E. L. Belzile $(\bowtie) \cdot$ M. Angers · M. Bédard Department of Surgery, Division of Orthopaedic Surgery, CHU de Québec-Université Laval, Quebec City, QC, Canada e-mail: etienne.belzile@chudequebec.ca; michele.angers.1@ulaval.ca and custom implants led to the development of a unique UKA implant system. Custom UKA would offer clinician the opportunity to accurately restore each patient's femoral and tibial morphology while providing additive material to compensate for cartilage loss. Using this particular technique, better restoration of the natural knee kinematic is achieved through personalization of the implants.

Current modern off-the-shelf (OTS) UKA systems propose a joint mechanics based on standardized femoral and tibial morphologies of various sizes extracted from image banks obtained from cohort of normal knees. Most UKA designs have idealized or simplified knee joint biomechanics for the medial compartment. The surgical technique dictates to properly position that implant for optimal function on the medial or lateral compartment. Early results have shown $85 \%$ to $98 \%$ revisionfree joint survival at 10 years [1-4]. Systematic reviews failed to show dominance of one design over another and rather promote equivalent clinical function and risk of revision surgery [5, 6]. Modern reports of fixed bearing devices account for similar implant survival rates $[7,8]$.

Despite attempts at summarizing femoral condyle morphology into a simple geometrical shape, one has to recognize that the medial and lateral condyles have different morphologies in terms of radius of curvature, $\mathrm{j}$-curve definition and condyle width [9-11]. These differences may not be fully accommodated for by existing OTS implants [12]. The inventory requirements necessary to 
cover all possible morphology or variability would be tremendous and therefore impossible to obtain. Instead, minor differences in femoral or tibial morphologies are averaged or ignored by the implant designers. In an era of increasing precision, patient-specific implants have shown statistically superior bone coverage [12]. Using statistical shape models, an estimate of what represents healthy distal femur has been recently conducted [13]. Patient-specific UKA have also been developed [14] and put to clinical use [15].

Why should we be looking for solutions? The most common reasons for failure of UKA today beside infections are polyethylene wear $[6,16]$, osteoarthritis progression and aseptic loosening [17, 18]. Malposition of implants has been identified as the most important cause leading to aseptic loosening in 559 UKAs with a survival of $83.7 \pm 3.5 \%$ at 10 years in a multicentric retrospective study [19]. They concluded that a joint space height $>2 \mathrm{~mm}$, tibial component obliquity $>3^{\circ}$, a tibial slope value $>5^{\circ}$ or a change in slope $>2^{\circ}$ and $>6^{\circ}$ divergence between the tibial and femoral components decreased significantly the prosthesis survival rate.

\subsection{What Is the Rational for Custom UKA?}

Most patients with medial knee osteoarthritis have a physiological varus aligned morphology before developing further degenerative changes. Thus, it is probably unnecessary to modify this alignment to neutral postoperatively. The custom design aims at $2^{\circ}$ to $3^{\circ}$ of hip-knee-ankle angle after implantation for a medial UKA [20-22].

The custom design software ensures proper preoperative planning of implant position which has been shown to be capital to optimize femoral to tibial contact stress area [23]. One may have the advantage, during preoperative planning, to measure and adjust such contact stress area. Furthermore, the height of the prosthetic joint space can also be built in the design of the components. This latter option allows implants to be seated at or less than $1 \mathrm{~mm}$ below the lateral compartment cartilage height [11] and avoids differences greater than $2 \mathrm{~mm}$ which have been shown to be detrimental to implant survival [19]. The surgeon should aim to use a $7 \mathrm{~mm}$ or $8 \mathrm{~mm}$ tibial polyethylene insert upon final implant cementation [16]. Using personalized cutting blocks, bone cut height can be accurately predicted and performed according to the optimal preoperative planning.

\subsection{Which Problems Does It Solve?}

Two problems are addressed by the custom UKA implant strategy. First, the exact reproduction of the patient's femoral condyle curvature and morphology combined with the natural patient's tibial slope should replicate native ligamentous tension throughout the full range of motion. Indeed, accurate ligamentous laxities across the full knee range of motion remains a challenge for the surgeon. A personalized knee implant design may offer a complete strategy to reach complete stress-free ligamentous range of motion across all variability in knee anatomy [24].

Secondly, precision in bone preparation is capital in order to position the implants according to the planned and measured location. Complications in implant positioning should be avoidable by improved instrumentation and surgical guidance. Obtaining proper implant positioning along with native morphological articular reconstruction will most likely lead to decreased premature implant loosening [14] and enhance gait pattern normalization [25].

\subsection{For Who (Best Indications)?}

Any patients presenting with unicompartmental knee pathology with intact cruciate and collateral ligaments would be ideal candidate to undergo custom UKA. Classic indications for OTS UKA have been well established [26], and custom UKA does not differ from these guidelines. Proper patient selection remains the best predictor of success for this type of surgery. Of mention, important osteonecrosis jeopardizing implant fixation, 
local malignancy, active infection, inflammatory arthritis, limited preoperative ROM, deformities greater than $10^{\circ}$, and more than $5 \mathrm{~mm}$ of unipolar bone defect would represent contraindications to this particular procedure.

\subsection{What Is the Process?}

After the traditional clinical encounter with a patient's history and complete physical exam, weight-bearing long-leg anteroposterior (AP) radiographs are obtained to evaluate the extent of the joint space narrowing and the femoro-tibial mechanical alignment. Once the patient is confirmed as a good candidate for a custom UKA, additional AP valgus stress views of the knee in extension are obtained [27]. This manoeuvre provides information on the unaffected compartment [28] and ensures relative quantification of the affected joint space gap when the collateral ligament is under full tension.

The patient is then required to undergo $\mathrm{CT}$ or MRI imaging of the affected knee. Images are converted to $3 \mathrm{D}$ volumes, and customization of the implant is prepared based on proprietary guidelines. Patient-specific instrumentation in the form of 3D-printed cutting guides for both the femur and tibia are then produced for every case. The femoral and tibial implants are manufactured according to the preoperative plan established by the surgeon in combination with a technician and an engineer. The treating physician has to accept the final preoperative planning before the custom cutting guides and implants can be manufactured. Polyethylene liners are produced in different thicknesses within a range of 5-9 $\mathrm{mm}$. All components and cutting guides are made available between 2 and 6 weeks following the patient imagery and implant prescription.

\subsection{Clinical Evidence Supporting This Concept?}

The current literature regarding personalized UKA components is relatively scarce since this concept is emerging. Multiple studies are avail- able on OTS UKA and show excellent 10- to 15-year survivorship [7, 8, 29]. Gait analysis following OTS UKA has shown a closer-to-normal gait restoration with UKA when compared to total knee arthroplasty (TKA) [30]. Unfortunately, OTS UKA do not necessarily restore normal gait patterns [25]. Customized 3D-printed UKA implants restore the normal knee anatomy and theoretically could restore normal gait patterns since physiological ligamentous tension should be obtained throughout the range of motion.

Researchers have worked with patient-specific instrumentation (PSI) for UKA implantation and have found $3.3 \%$ tibial fractures with $16.4 \%$ sagittal plane outliers [31]. While some authors propose no benefits $[32,33]$, others have demonstrated marked improvements with PSI [34]. Specifically relating to the BUKS ${ }^{\mathrm{TM}}$ (Bodycad, QC, Canada) custom UKA design, early reports are promising [35]. Since most of the technical errors in surgery are surgeon related [36], and surgical experience is capital in UKA surgical technique [37, 38], PSI may represent as an important tool for the inexperienced surgeon $[39,40]$.

\subsection{What Is Its Cost-Effectiveness?}

There are no published studies on custom UKA and no cost-effectiveness studies of PSI for UKA.

\subsection{Clinical Case Presentation}

A 56-year-old man presents with left knee pain and limping at activity. The patient evolved well after undergoing sessions of physical therapy and the use of some oral NSAIDs. He later developed progressive pain after prolonged walking and long days standing still. The patient is comfortable in the sitting position but reports having sharp pains in transitioning from the sitting to a standing position.

On physical exam, the patient displays a leftsided prolonged weight-bearing limp without a varus thrust. Clinically measured leg length is equal. Lower leg muscle strength is within 



Fig. 19.1 Radiographic imaging confirms a joint space narrowing as shown medially

normal. Range of motion of the left hip shows pain-free and supple full range of motion. The left knee displays flexion from $5^{\circ}$ to $110^{\circ}$. A valgus stress view at $0^{\circ}$ of flexion demonstrated a $5 \mathrm{~mm}$ medial gap opening. All other ligamentous tests are within normal limits with a firm end feel.

\subsection{Preoperative Radiographs}

Radiographic imaging (Fig. 19.1) confirms a joint space narrowing as shown medially. Fulllength films and stress view in valgus complete preoperative planning (Fig. 19.2).

\subsection{Surgical Details}

Similar to conventional UKA, a standard 8-12 cm skin incision is performed along the medial border of the patellar tendon (Fig. 19.3a). A quadriceps-sparing minimally invasive medial parapatellar arthrotomy is favoured in order to obtain adequate exposure to the medial compartment of the knee (Fig. 19.3b). Although the decision to perform a minimally invasive incision to limit soft-tissue trauma is the surgeon's own prerogative, this procedure can easily be performed using both the standard and minimally invasive approach.

The surgical technique first requires the subperiosteal exposure of the antero-medial proximal aspect of the tibial to allow adequate sitting of the patient-specific 3D-printed nylon cutting guides. Failure to obtain perfect sitting of the cutting block will result in malpositioning of the device and therefore produce inadequate and/or misaligned bone resection. The surgeon should not hesitate to obtain better exposure through a longer skin incision if perfect placement of the cutting block cannot be confirmed using the standard approach. The cutting block position should be assessed using the dentist hook to ensure that the periphery of the guide perfectly sits on the bone and that no voids are palpated. Once adequate positioning is confirmed, the cutting block can be secured to bone using two or three small $3.5 \mathrm{~mm}$ cortical screws (Fig. 19.3c).

Tibial bone resection can then be safely performed using a drill and making sure to drill every hole provided in the cutting guide (Fig. 19.3d) To enhance stability of the cutting block, a first drill bit can remain through the first drill hole, and the remaining holes can be drilled using a second drill bit. After drilling every holes, the cutting block can be fragmented using a cutter (Fig. 19.3e), and the remaining axial and sagittal 
Fig. 19.2 Full-length films and stress view in valgus complete preoperative planning
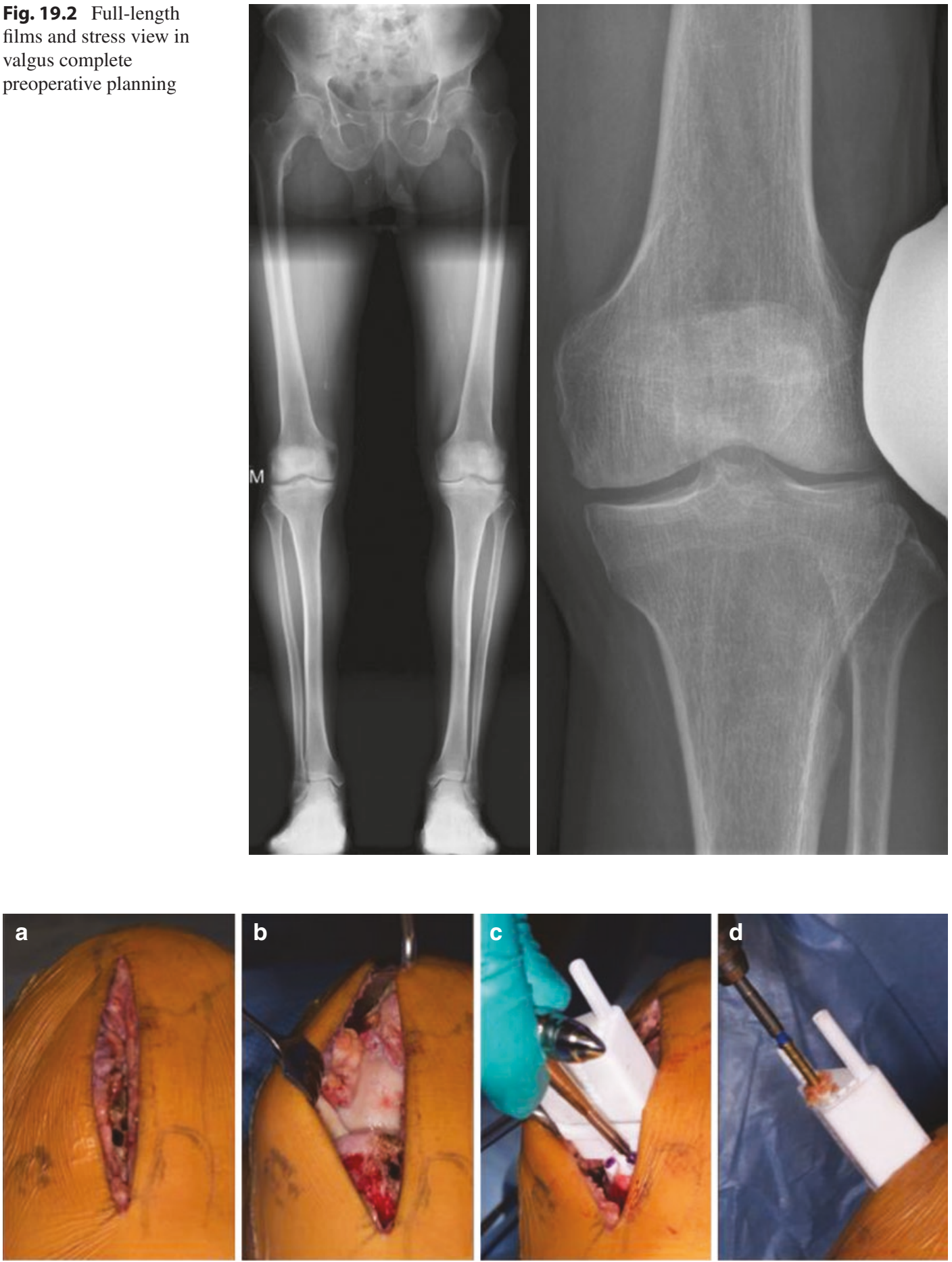

Fig. 19.3 Tibial bone resection starts with a medial incision on the left knee (a), medial arthrotomy (b), then the tibial cutting guide is stabilized with screws (c), bone is drilled (d), cutting guide is dismantled (e), a vertical pass

is performed with a graduated osteotome (f), a horizontal pass with the osteotome, (g) and the cutting guide is removed $(\mathbf{h})$ 

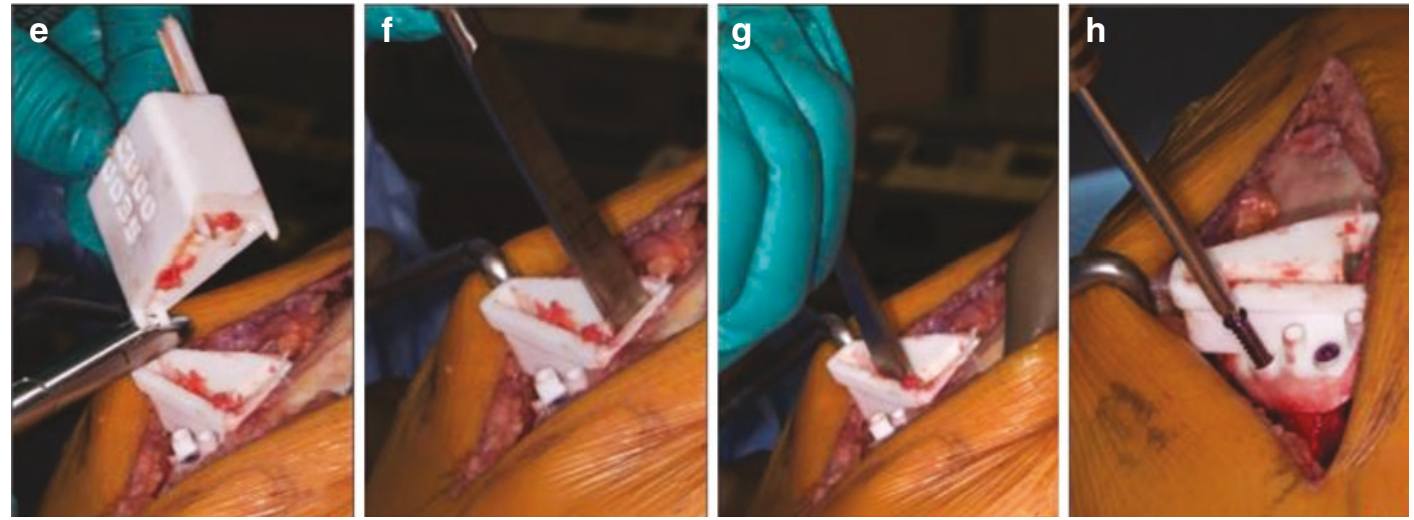

Fig. 19.3 (continued)
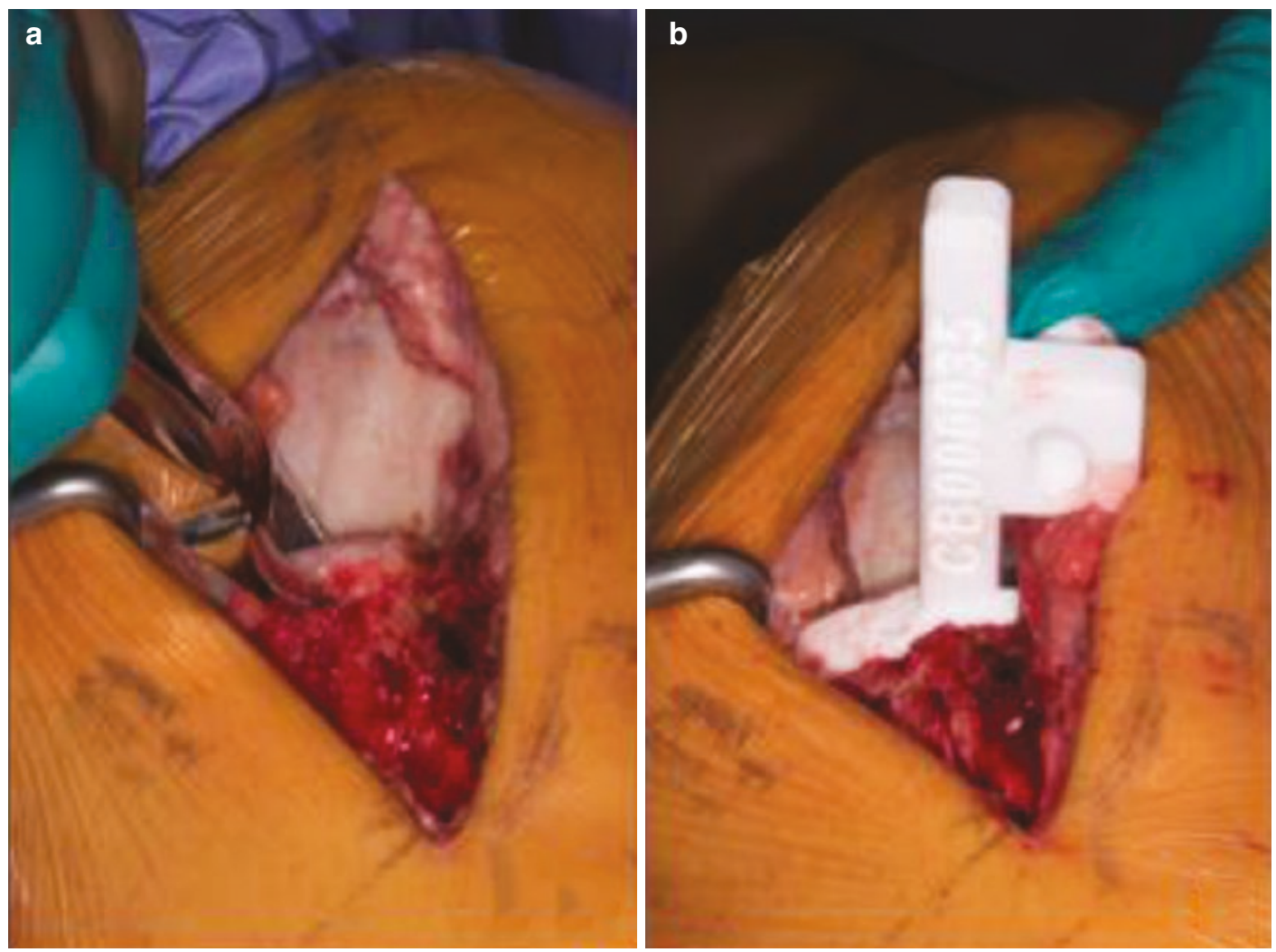

Fig. 19.4 The tibial bone is removed, (a) and a validator is used to verify resection (b)

tibial bone cuts can be completed using a graduated straight osteotome (Fig. 19.3f, g) The tibial cutting block is discarded after removing the screws (Fig. 19.3h).

The resected tibial bone is then removed using a grasping instrument (Fig. 19.4a). A tibial bone cut validator, provided for each case, is then placed on the proximal tibia in order to ensure that the amount of resected tibial bone matches the preoperative planned resection (Fig. 19.4b). The handle of the tibial validator also features an alignment hole allowing the surgeon to use 
a standard drop rod to validate tibial bone cut alignment if desired.

As for the femoral preparation, a custom femoral cutting block is required but should sit directly on hard sclerotic bone of the condyle. Therefore, a bone curette is used to scrape off any remaining cartilage left on the medial femoral condyle. Proper positioning of the femoral cutting block should also be assessed using a dentist hook, making sure once again that the periphery of the cutting block sits perfectly on the bone and that no voids are left underneath the cutting block.

The femoral cutting block is then secured to bone using $3.5 \mathrm{~mm}$ cortical screws (Fig. 19.5a). Sequential drilling (Fig. 19.5b), fragmentation of the cutting block (Fig. 19.5c), and osteotome passes (Fig. 19.5d) can be performed similarly to the tibial bone resection. After removal of the femoral cutting block, a nylon femoral component trial along with the tibial bone resection validator is inserted, and the femoral side is fixed with
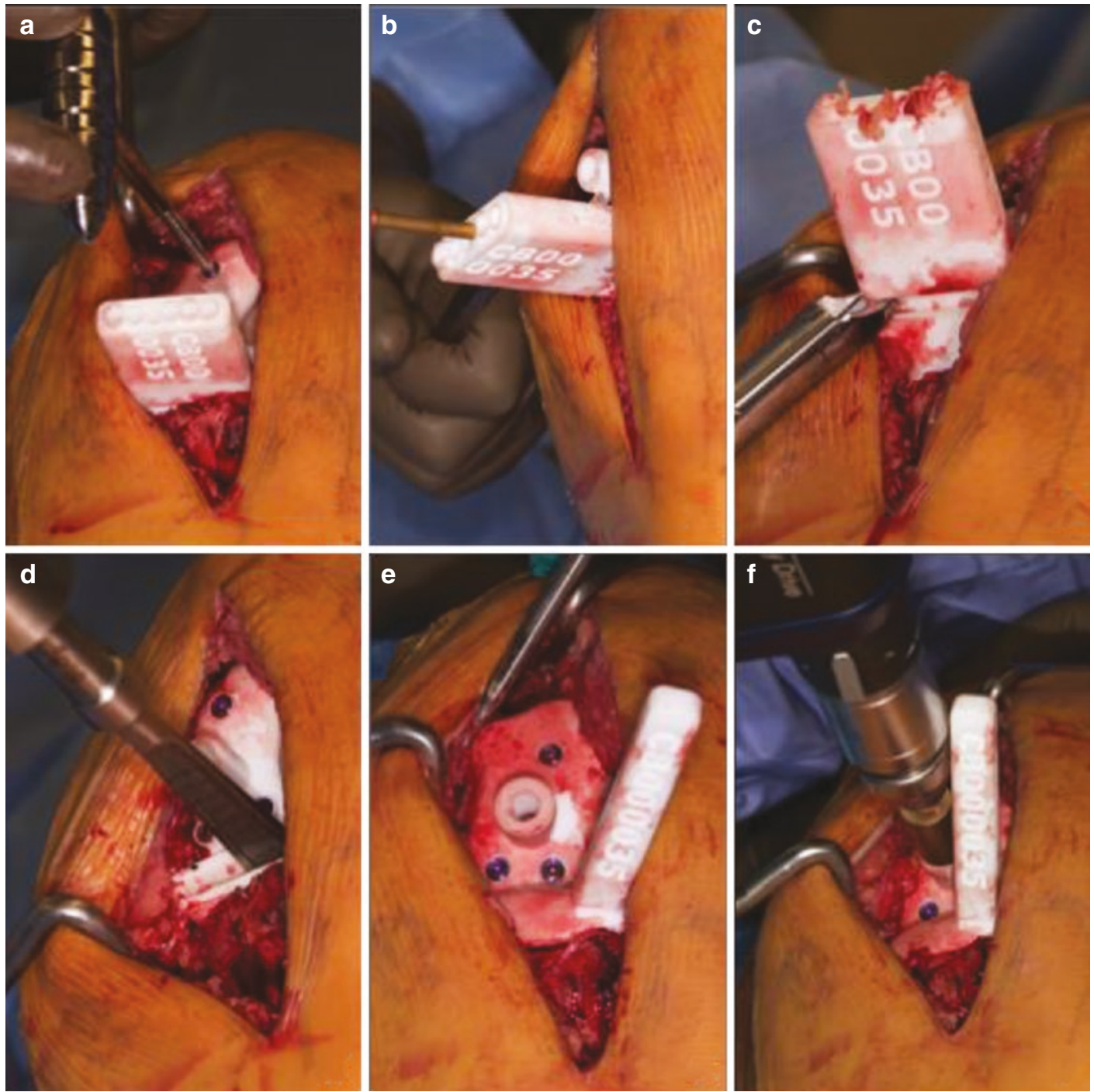

Fig. 19.5 Femoral bone resection starts by stabilizing the femoral cutting guide with screws (a) on exposed subchondral bone, bone is drilled (b), cutting guide is dis- mantled (c), passes are performed with a graduated osteotome (d), and the central peg hole is drilled (e, f) as the tibial bone resection validator is left in situ 
screws. This allows for validation of the femoral component position before reaming of the peg hole using the provided femoral reamer through the base of the femoral trial (Fig. 19.5e, f).

After completing the bone resections, the surgeon should be able to proceed with trialing using the 3D-printed nylon components (Fig. 19.6a). At this stage, the proper polyethylene thickness should be assessed making sure ligamentous stability is perfectly achieved throughout the full range of motion. It is always preferable to leave a physiologic $2 \mathrm{~mm}$ medial compartment laxity to avoid overtensioning of the medial compartment and potentially inducing a mechanically valgusaligned medial arthroplasty.

After completing trials and cleaning of bony surfaces, cementation and impaction of the final tibial implant are performed in a routine fashion. Excess cement is removed, and a $3.5 \mathrm{~mm}$ cor- tical bone screw is inserted in order to help in proper component positioning. The final femoral component is then cemented and impacted. Once again, one $4.0 \mathrm{~mm}$ cortical bone screw helps enhancing the final position of the femoral implant. Polyethylene thickness trialing can be repeated (Fig. 19.6b).

The final polyethylene is then inserted (Fig. 19.6c) and locked into the tibial baseplate using the provided locking pin (Fig. 19.6d). When cement has fully hardened, complete physical examination of the knee should be done to confirm full range of motion, ligamentous stability, proper patellar tracking, and the absence of soft-tissue impingement. Finally, the arthrotomy is closed using 1-0 absorbable sutures. Subcutaneous tissue is closed in a subcuticular fashion using 2-0 sutures. A sterile dressing is applied. Patient underwent an uneventful postoperative recovery (Fig. 19.7).
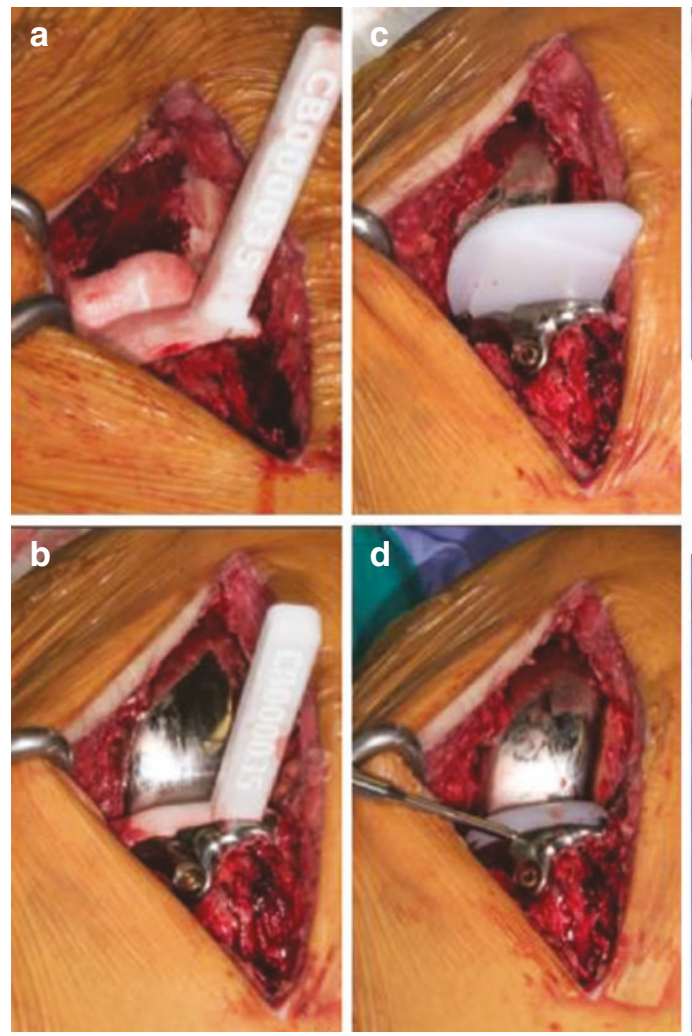

Fig. 19.6 Before the final implantation, 3D-printed nylon trial components are used for trialing (a), once implants are cemented, different thicknesses of polyethylene liners are trialed for proper ligament tensioning (b),



and final liner is inserted and locked in place (c, d). Fullsize 3D models are provided with the custom implants to allow for studying the implant position, size, and fit (e-g) 


\subsection{Follow-up Radiographs}
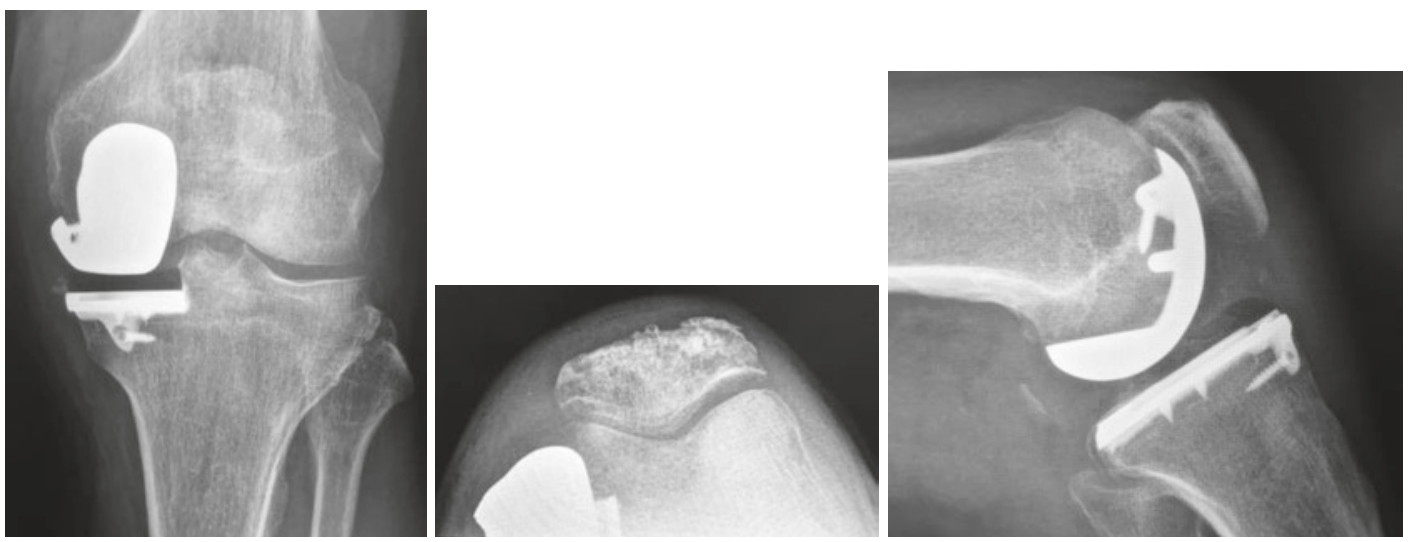

Fig. 19.7 Six months postoperative radiographs

\subsection{Indications and Contraindications}

\subsubsection{Indications}

- Medial osteoarthritis of the knee

- $<15^{\circ}$ coronal malalignment

- Efficient anterior cruciate ligament

- Flexion contracture $<15^{\circ}$ (debatable [41])

\subsubsection{Contraindications}

- Tricompartmental knee osteoarthritis

- Unstable knee

- Femoral condyle osteonecrosis

\subsection{Conclusion}

The next decade of knee implant designs will develop with amazing new technologies including navigation, robotics, and virtual reality. The innovating custom design UKA presented in this chapter shows promising strategies, exploiting knowledge from past designs and positioning its expected development in line with existing variability in functional knee phenotypes. Further adjustment in UKA custom designs may have to be aligned with clinical trial findings and implant biomechanical tests. The clinician has to remain vigilant about new technologies but critic in its introduction and initial use.

\section{References}

1. Murray DW, Goodfellow JW, O'Connor JJ. The Oxford medial unicompartmental arthroplasty: a ten-year survival study. J Bone Jt Surg. 1998;80-B(6):983-9.

2. Capra SW, Fehring TK. Unicondylar arthroplasty. A survivorship analysis. J Arthroplasty. 1992;7(3):247-51.

3. Berger RA, Nedeff DD, Barden RM, Sheinkop MM, Jacobs JJ, Rosenberg AG, et al. Unicompartmental knee arthroplasty. Clinical experience at 6to 10-year followup. Clin Orthop Relat Res. 1999;(367):50-60.

4. Scott RD, Cobb AG, McQueary FG, Thornhill TS. Unicompartmental knee arthroplasty. Eight- to 12-year follow-up evaluation with survivorship analysis. Clin Orthop Relat Res. 1991;(271):96-100.

5. Peersman G, Stuyts B, Vandenlangenbergh T, Cartier P, Fennema P. Fixed- versus mobile-bearing UKA: a systematic review and meta-analysis. Knee Surg Sports Traumatol Arthrosc. 2015;23(11):3296-305.

6. Ko Y-B, Gujarathi MR, Oh K-J. Outcome of unicompartmental knee arthroplasty: a systematic review of comparative studies between fixed and mobile bearings focusing on complications. Knee Surg Relat Res. 2015;27(3):141-8.

7. Panni AS, Vasso M, Cerciello S, Felici A. Unicompartmental knee replacement provides early clinical and functional improvement stabilizing over time. Knee Surg Sports Traumatol Arthrosc. 2012;20(3):579-85. 
8. Foran JRH, Brown NM, Valle Della CJ, Berger RA, Galante JO. Long-term survivorship and failure modes of unicompartmental knee arthroplasty. Clin Orthop Relat Res. 2013;471(1):102-8.

9. Nuño N, Ahmed AM. Sagittal profile of the femoral condyles and its application to femorotibial contact analysis. J Biomech Eng. 2001;123(1):18-26.

10. Zoghi M, Hefzy MS, Fu KC, Jackson WT. A threedimensional morphometrical study of the distal human femur. Proc Inst Mech Eng H. 1992;206(3):147-57.

11. Du PZ, Markolf KL, Levine BD, McAllister DR, Jones KJ. Differences in the radius of curvature between femoral condyles: implications for osteochondral allograft matching. J Bone Jt Surg. 2018;100-A(15):1326-31.

12. Carpenter DP, Holmberg RR, Quartulli MJ, Barnes CL. Tibial plateau coverage in UKA: a comparison of patient specific and off-the-shelf implants. J Arthroplasty. 2014;29(9):1694-8.

13. van der Merwe J, van den Heever DJ, Erasmus PJ. Estimating regions of interest on the distal femur. Med Eng Phys. 2018;60:23-9.

14. Harrysson OLA, Hosni YA, Nayfeh JF. Customdesigned orthopedic implants evaluated using finite element analysis of patient-specific computed tomography data: femoral-component case study. BMC Musculoskelet Disord. 2007;8:91.

15. Fitz W. Unicompartmental knee arthroplasty with use of novel patient-specific resurfacing implants and personalized jigs. J Bone Jt Surg. 2009;91-A(Suppl 1):69-76.

16. Parratte S, Argenson J-NA, Pearce O, Pauly V, Auquier P, Aubaniac J-M. Medial unicompartmental knee replacement in the under-50s. J Bone Jt Surg. 2009;91-B(3):351-6.

17. Liddle AD, Pandit H, O’Brien S, Doran E, Penny ID, Hooper GJ, et al. Cementless fixation in Oxford unicompartmental knee replacement: a multicentre study of 1000 knees. Bone Jt J. 2013;95-B(2):181-7.

18. Pandit H, Liddle AD, Kendrick BJL, Jenkins C, Price AJ, Gill HS, et al. Improved fixation in cementless unicompartmental knee replacement: five-year results of a randomized controlled trial. J Bone Jt Surg. 2013;95-A(15):1365-72.

19. Chatellard R, Sauleau V, Colmar M, Robert H, Raynaud G, Brilhault J, et al. Medial unicompartmental knee arthroplasty: does tibial component position influence clinical outcomes and arthroplasty survival? Orthop Traumatol Surg Res. 2013;99(4 Suppl):S219-25.

20. Bellemans J, Colyn W, Vandenneucker H, Victor J. The Chitranjan Ranawat award: is neutral mechanical alignment normal for all patients? The concept of constitutional varus. Clin Orthop Relat Res. 2012;470(1):45-53.

21. Eckhoff DG, Bach JM, Spitzer VM, Reinig KD, Bagur MM, Baldini TH, et al. Three-dimensional mechanics, kinematics, and morphology of the knee viewed in virtual reality. J Bone Jt Surg. 2005;87-A(Suppl 2):71-80.

22. Mullaji AB, Shah S, Shetty GM. Mobile-bearing medial unicompartmental knee arthroplasty restores limb alignment comparable to that of the unaffected contralateral limb. Acta Orthop. 2017;88(1):70-4.

23. Diezi C, Wirth S, Meyer DC, Koch PP. Effect of femoral to tibial varus mismatch on the contact area of unicondylar knee prostheses. Knee. 2010;17(5):350-5.

24. Hirschmann MT, Behrend H. Functional knee phenotypes: a call for a more personalised and individualised approach to total knee arthroplasty? Knee Surg Sports Traumatol Arthrosc. 2018;26(10):2873-4.

25. Kim M-K. Unicompartmental knee arthroplasty fails to completely restore normal gait patterns during level walking. Knee Surg Sports Traumatol Arthrosc. 2018;26(11):3280-9.

26. Kozinn SC, Scott R. Unicondylar knee arthroplasty. J Bone Jt Surg. 1989;71-A(1):145-50.

27. Eriksson K, Sadr-Azodi O, Singh C, Osti L, Bartlett J. Stress radiography for osteoarthritis of the knee: a new technique. Knee Surg Sports Traumatol Arthrosc. 2010;18(10):1356-9.

28. Bergeson AG, Berend KR, Lombardi AV, Hurst JM, Morris MJ, Sneller MA. Medial mobile bearing unicompartmental knee arthroplasty: early survivorship and analysis of failures in 1000 consecutive cases. J Arthroplasty. 2013;28(9 Suppl):172-5.

29. Saragaglia D, Bevand A, International RR. Results with nine years mean follow up on one hundred and three $\mathrm{KAPS}^{\circledR}$ uni knee arthroplasties: eighty six medial and seventeen lateral. Eur J Orthop Surg Traumatol. 2018;42(5):1061-6.

30. Jones GG, Kotti M, Wiik AV, Collins R, Brevadt MJ, Strachan RK, et al. Gait comparison of unicompartmental and total knee arthroplasties with healthy controls. Bone Jt J. 2016;10(Suppl B):16-21.

31. Leenders AM. A high rate of tibial plateau fractures after early experience with patient-specific instrumentation for unicompartmental knee arthroplasties. Knee Surg Sports Traumatol Arthrosc. 2018;26(11): 3491-8.

32. Ollivier M, Parratte S, Lunebourg A, Viehweger E, Argenson J-N. The John Insall award: no functional benefit after unicompartmental knee arthroplasty performed with patient-specific instrumentation: a randomized trial. Clin Orthop Relat Res. 2016;474(1):60-8.

33. Alvand A, Khan T, Jenkins C, Rees JL, Jackson WF, Dodd CAF, et al. The impact of patient-specific instrumentation on unicompartmental knee arthroplasty: a prospective randomised controlled study. Knee Surg Sports Traumatol Arthrosc. 2018;26(6):1662-70.

34. Dao Trong ML, Diezi C, Goerres G, Helmy N. Improved positioning of the tibial component in unicompartmental knee arthroplasty with patientspecific cutting blocks. Knee Surg Sports Traumatol Arthrosc. 2015;23(7):1993-8. 
35. Belzile E, Rivet-Sabourin G, Bédard M, Robichaud H, Angers M, Bédard M. Évaluation de la précision d'implantation d'une prothèse unicompartimentale de genou utilisant un guide de coupe personnalisé. Orthop Traumatol Surg Res. 2017;103S:S31.

36. Vasso M, Antoniadis A, Helmy N. Update on unicompartmental knee arthroplasty: current indications and failure modes. EFORT Open Rev. 2018;3(8):442-8.

37. Liddle AD, Pandit H, Judge A, Murray DW. Effect of surgical caseload on revision rate following total and unicompartmental knee replacement. J Bone Jt Surg. 2016;98-A(1):1-8.

38. Zambianchi F, Digennaro V, Giorgini A, Grandi G, Fiacchi F, Mugnai R, et al. Surgeon's experience influences UKA survivorship: a comparative study between all-poly and metal back designs. Knee Surg Sports Traumatol Arthrosc. 2015;23(7):2074-80.

39. Jones GG, Logishetty K, Clarke S, Collins R, Jaere $\mathrm{M}$, Harris S, et al. Do patient-specific instruments (PSI) for UKA allow non-expert surgeons to achieve the same saw cut accuracy as expert surgeons? Arch Orthop Trauma Surg. 2018;138(11):1601-8.

40. Schotanus MGM, Thijs E, Heijmans M, Vos R, Kort NP. Favourable alignment outcomes with MRIbased patient-specific instruments in total knee arthroplasty. Knee Surg Sports Traumatol Arthrosc. 2018;26(9):2659-68.

41. Purcell RL, Cody JP, Ammeen DJ, Goyal N, Engh GA. Elimination of preoperative flexion contracture as a contraindication for unicompartmental knee arthroplasty. J Am Acad Orthop Surg. 2018;26(7):e158-63.

Open Access This chapter is licensed under the terms of the Creative Commons Attribution 4.0 International License (http://creativecommons.org/licenses/by/4.0/), which permits use, sharing, adaptation, distribution and reproduction in any medium or format, as long as you give appropriate credit to the original author(s) and the source, provide a link to the Creative Commons license and indicate if changes were made.

The images or other third party material in this chapter are included in the chapter's Creative Commons license, unless indicated otherwise in a credit line to the material. If material is not included in the chapter's Creative Commons license and your intended use is not permitted by statutory regulation or exceeds the permitted use, you will need to obtain permission directly from the copyright holder. 